\title{
Sonar Inside Your Body: Prototyping Ultrasonic Intra-body Sensor Networks
}

\author{
G. Enrico Santagati and Tommaso Melodia \\ University at Buffalo, The State University of New York, New York, USA \\ \{santagat, tmelodia\}@buffalo.edu
}

\begin{abstract}
Biomedical systems of implanted miniaturized sensors and actuators interconnected into an intra-body area network could enable revolutionary healthcare and clinical applications. Given the well-understood limitations of radio frequency (RF) propagation in the human body, in our previous work we investigated the use of ultrasonic waves as an alternative physical carrier of information [1], and proposed Ultrasonic WideBand (UsWB), an ultrasonic multipath-resilient integrated physical and medium access control (MAC) layer protocol [2]. In this paper, we discuss the design and implementation of a software-defined testbed architecture for ultrasonic intra-body area networks, and propose the first experimental demonstration of the feasibility of ultrasonic communications in biological tissues. We first discuss in detail our FPGA-based prototype implementation of UsWB. We then demonstrate how the prototype can flexibly trade performance off for power consumption, and achieve, for bit error rates (BER) no higher than $10^{-6}$, either (i) high-data rate transmissions up to $700 \mathrm{kbit} / \mathrm{s}$ at a transmit power of $-14 \mathrm{dBm}$ $(\approx 40 \mu \mathrm{W})$, or (ii) low-data rate and lower-power transmissions down to $-21 \mathrm{dBm}(\approx 8 \mu \mathrm{W})$ at $70 \mathrm{kbit} / \mathrm{s}$. Finally, we show how the UsWB MAC protocol allows multiple transmitter-receiver pairs to coexist and dynamically adapt the transmission rate according to channel and interference conditions to maximize throughput while satisfying predefined reliability constraints.
\end{abstract}

\section{INTRODUCTION}

Biomedical systems of implanted or wearable miniaturized sensors and actuators wirelessly interconnected into an intrabody area network could enable revolutionary healthcare and clinical applications [3]. For example, remote cardiac rhythm monitors could detect cardiovascular malfunctions and trigger preemptive measures to mitigate their effects. Continuous glucose monitoring in diabetic patients could enable reactive administration of insulin through under-skin miniaturized drug deliverers; while pill-sized ingestible cameras could enable remote monitoring of the digestive tract and avoid intrusive examination techniques such as gastroscopy.

While application-specific implantable medical microsystems at the $s u b-m m^{3}$ scale with perpetual energy harvesting have been demonstrated [4], the underlying root challenge of enabling wireless networks of intra-body miniaturized sensors and actuators that communicate through body tissues is substantially unaddressed. The main obstacle to enabling this vision of networked implantable devices is posed by the dielectric nature of the human body, which is composed

This material is based upon work supported by the National Science Foundation under grant CAREER CNS-1253309.

978-1-4799-3360-0/14/\$31.00 (C)2014 IEEE primarily $(65 \%)$ of water, a medium through which radio frequency $(\mathrm{RF})$ electromagnetic waves do not easily propagate. In addition, the medical community is still divided on the real risks caused by exposure to $\mathrm{RF}$ radiations.

Given the limitations of RF propagation, in [1], [2] we proposed and investigated the use of ultrasonic waves as an alternative carrier of information in human tissues. Lowfrequency acoustic waves (up to $100 \mathrm{kHz}$ ) have found application in underwater communications [5], while ultrasounds at higher frequencies are extensively used in medical imaging applications [6]. Innovations in piezoelectric materials and fabrication methods have made miniaturized transducers, at the micro [7] and even nano scales [8] a reality. Moreover, the medical experience of the last decades has demonstrated that ultrasonic heat dissipation in tissues is minimal compared to RF [9], and that ultrasounds are fundamentally safe, as long as acoustic power dissipation in tissue is limited to specific safety levels [10], [1].

In [1], we showed that intra-body ultrasound propagation is severely affected by multipath caused by inhomogeneity of the body in terms of density, sound speed, and the pervasive presence of small organs and particles. Based on these observations, in [2], we proposed Ultrasonic WideBand (UsWB), a new ultrasonic multipath-resilient physical and medium access control (MAC) layer integrated protocol. UsWB is based on the idea of transmitting short carrierless ultrasonic pulses following a pseudo-random adaptive time-hopping pattern, with a superimposed adaptive spreading code. Impulsive transmission and spread-spectrum encoding combat the effects of multipath and scattering and introduce waveform diversity among interfering nodes so that multiple users can coexist with limited interference on the same channel. However, as of today, there have been no attempts at experimentally demonstrating ultrasonic communications through body tissues.

In this paper, we make the following core contributions: (i) we present the design and implementation of a softwaredefined testbed architecture for ultrasonic intra-body area networks. The testbed consists of software-defined nodes communicating via ultrasonic waves through media that emulate acoustic propagation through biological tissues with high fidelity, i.e., ultrasonic phantoms; (ii) we experimentally demonstrate the feasibility of ultrasonic communications in human tissues. To this purpose, we design an FPGAbased prototype implementation of the UsWB physical and medium access control protocols and evaluate extensively its 
performance through a human-kidney phantom. We show that our prototype can flexibly trade data rate performance for power consumption, and achieve, for bit error rates (BER) no higher than $10^{-6}$, either (i) high-data rate transmissions up to $700 \mathrm{kbit} / \mathrm{s}$ at a transmit power of $-14 \mathrm{dBm}(\approx 40 \mu \mathrm{W})$, or (ii) low-data rate and lower-power transmissions down to $-21 \mathrm{dBm}(\approx 8 \mu \mathrm{W})$ at $70 \mathrm{kbit} / \mathrm{s}$ (in addition to numerous intermediate configurations). Moreover, we show how the UsWB MAC protocol allows multiple concurrent users to coexist and dynamically adapt their transmission rate to channel and interference conditions to maximize throughput while satisfying predefined reliability constraints, e.g., maximum packet drop rate.

The remainder of the paper is organized as follows. In Section II we briefly discuss basic aspects of ultrasonic intrabody communications and introduce the UsWB transmission and medium access technique. In Section III we present the proposed testbed architecture. In Section IV we discuss the communication system architecture and implementation while in Section V we discuss details of the FPGA implementation of transmitter and receiver. In Sections VI we extensively evaluate the performance of UsWB. Finally, in Section VII we conclude the paper.

\section{BACKGROUND}

\section{A. Ultrasonic Intra-Body Communications}

Ultrasounds are mechanical waves that propagate in an elastic medium at frequencies above the upper limit for human hearing, i.e., $20 \mathrm{kHz}$.

Attenuation. Two main mechanisms contribute to ultrasound attenuation in tissues, i.e., absorption and scattering. An initial pressure $P_{0}$ decays at a distance $d$ according to [11]

$$
P(d)=P_{0} e^{-\alpha d},
$$

where $\alpha$ (in $\left[\mathrm{Np} \cdot \mathrm{cm}^{-1}\right]$ ) is an amplitude attenuation coefficient that captures all the effects that cause dissipation of energy from the ultrasound wave. Parameter $\alpha$ depends on the carrier frequency through $\alpha=a f^{b}$, where $f$ represents the carrier frequency (in $\mathrm{MHz}$ ) and $a$ (in $\left[\mathrm{Np} \mathrm{m}^{-1} \mathrm{MHz}^{-\mathrm{b}}\right]$ ) and $b$ are attenuation parameters characterizing the tissue [1].

Propagation Speed. Ultrasonic wave propagation is affected by propagation delays that are orders of magnitude higher than RF. The propagation speed of acoustic waves in biological tissues is approximately $1500 \mathrm{~m} / \mathrm{s}$, as compared to $2 \times 10^{8} \mathrm{~m} / \mathrm{s}$ [12] for RF waves.

Operating Frequency. Key considerations in determining the operating frequency are (i) the frequency dependence of the attenuation coefficient, and (ii) the frequency dependence of the beam spread of ultrasonic transducers (which is inversely proportional to the ratio of the diameter of the radiating surface and the wavelength [1]). Therefore, higher frequencies help keep the transducer size small, but result in higher signal attenuation. Since most biomedical sensing applications require directional transducers, one needs to operate at the lowest possible frequencies compatible with small-size transducers and required signal bandwidth. In [1], we showed that for

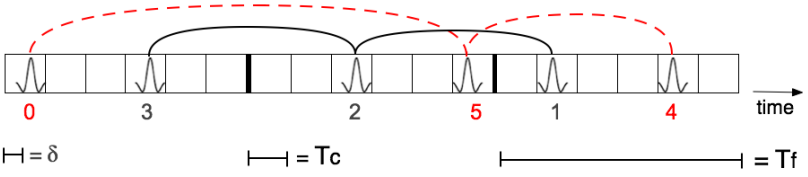

Fig. 1. Two concurrent transmissions with $N_{h}=6, N_{s}=3$, time-hopping sequences $\{3,2,1\}$ and $\{0,5,4\}$ and spreading codes $\{1,1,-1\}$ and $\{1,-1,-1\}$.

propagation distances in the order of several $\mathrm{cm}$ the operating frequency should not exceed $10 \mathrm{MHz}$.

Reflections and Scattering. The human body is composed of different organs and tissues with different sizes, densities and sound speeds. Therefore, it can be modeled as an environment with pervasive presence of reflectors and scatterers. The direction and magnitude of the reflected wave depend on the orientation of the boundary surface and on the acoustic impedance of the tissues [1], while scattered reflections occur when an acoustic wave encounters an object that is relatively small with respect to its wavelength or a tissue with an irregular surface. Consequently, the received signal is obtained as the sum of numerous attenuated, possibly distorted, and delayed versions of the transmitted signal.

\section{B. Ultrasonic WideBand}

Based on these observations, in [2] we proposed Ultrasonic WideBand (UsWB), a new impulse-radio inspired ultrasonic transmission and multiple access technique based on the idea of transmitting short information-bearing carrierless ultrasonic pulses, following a pseudo-random adaptive time-hopping pattern with a superimposed spreading code of adaptive length. Impulsive transmission and spread-spectrum encoding combat the effects of multipath and scattering and introduce waveform diversity among interfering transmissions.

Physical Layer. Consider, as in Fig. 1, a slotted timeline divided in slots of duration $T_{c}$, with slots organized in frames of duration $T_{f}=N_{h} T_{c}$, where $N_{h}$ is the number of slots per frame. Each user transmits one pulse per frame in a slot determined by a pseudo-random time-hopping sequence. Information is carried through pulse position modulation (PPM), i.e., a ' 1 ' symbol is carried by a pulse delayed by a time $\delta$ with respect to the beginning of the slot, while a '-1' symbol begins with the slot. Since a single pulse may collide with pulses transmitted by other users with a probability that depends on the frame size $N_{h}$, we represent each information bit with pseudo-orthogonal spreading codes of variable length, $N_{s}$ because of (i) their excellent, and well-understood multiple access performance, (ii) limited computational complexity, and (iii) inherent resilience to multipath. The resulting transmitted signal for a symbol $d$ can be modeled as

$$
s(t)=\sum_{j=0}^{N_{s}-1} p\left(t-c_{j} T_{c}-j T_{f}-\frac{a_{j} d+1}{2} \delta\right)
$$

where $p(t)$ is the pulse shape, $\left\{c_{j}\right\}$ is the time-hopping sequence with $0 \leq c_{j} \leq N_{h}-1,\left\{a_{j}\right\}$ is the pseudo-orthogonal spreading code of $N_{s}$ chips with $a_{j} \in\{-1,1\}$, and $\delta$ is the PPM shift of a pulse representing a ' 1 ' chip. 


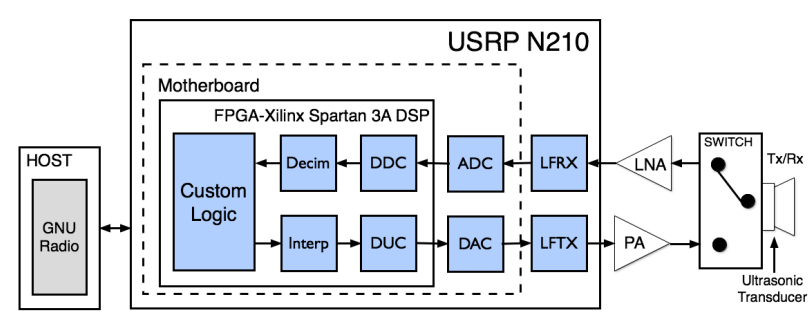

Fig. 2. Hardware architecture of an ultrasonic software-defined node.

Medium Access Control. The low-duty-cycle impulsebased transmission scheme with a superimposed spreading code allows multiple transmitters to coexist on the same channel. In UsWB, by dynamically and distributively adapting their time-hopping frame length and spreading code length, multiple users coexist without the need for mutual temporal exclusion between different transmissions (which is hard to achieve in ultrasonic channels affected by long propagation delays). By adapting frame and code length, users control the tradeoffs among (i) resilience to multi-user interference and ultrasonic channel errors, (ii) achievable information rate, and (iii) energy efficiency. As discussed in detail in [2], by controlling the time-hopping frame length $N_{h}$, i.e., the average inter-pulse time, a user can adapt the transmission rate (which decreases with larger time-hopping frame), and as a consequence modify the average radiated power and therefore the level of interference generated to other ongoing communications. By controlling $N_{s}$, i.e., the number of pulses per information bit, a user can control the tradeoff between robustness to multi-user interference and noise (which increases with longer spreading codes), energy consumption per bit (which increases linearly with increasing $N_{s}$ ) and information rate (decreasing with increasing $N_{s}$ ). UsWB optimally, distributively, and asynchronously regulates these tradeoffs to (i) maximize the communication rate, or (ii) minimize the energy consumption.

In this paper, we consider the rate-maximizing adaptation in [2], where each user distributively maximizes its transmission rate by selecting an optimal pair of code and frame lengths based on the current level of interference and channel quality for a given maximum tolerable BER. Rate adaptation is achieved through an ad-hoc designed protocol. A two-way handshake opens the connection between two nodes, $T_{x}$ and $R_{x} . T_{x}$ sends a Request-to-Transmit (R2T) packet to $R_{x}$. If $R_{x}$ is idle, a Clear-to-Transmit (C2T) packet is sent back to $T_{x}$. Once the connection has been established, the receiver $R_{x}$ estimates the interference and calculates the frame and spreading code lengths that maximize the communication throughput, as discussed in detail in [2]. This information is piggybacked into ACK or NACK packets.

\section{Ultrasonic Testbed Architecture}

We designed and implemented a reconfigurable platform to test ultrasonic communication and networking schemes. The testbed consists of ultrasonic software-defined nodes communicating through ultrasonic phantoms that emulate acoustic propagation through biological tissues with high fidelity. The proposed hardware architecture of an ultrasonic softwaredefined node is illustrated in Fig. 2. It consists of (i) a Universal Software Radio Peripheral (USRP) N210, (ii) a host machine, (iii) an electronic switch, (iv) an amplification stage, (v) and a high-frequency ultrasonic transducer.

USRP N210. Several Software Defined Radio (SDR) development platforms are available [13], [14] where FieldProgrammable Gate Arrays (FPGAs) or specialized processors are used for high-sample-rate digital signal processing. Among these, we selected USRP [13] because of its low cost and wide adoption in academia and industry. USRP N210 consists of a motherboard and two daughterboards. The motherboard is the main processing unit, and incorporates $\mathrm{AD} / \mathrm{DA}$ converters (a dual 100 MSPS 14-bit ADC and a dual 400 MSPS 16bit DAC), and an FPGA unit (Spartan 3A-DSP 3400). The daughterboards are RF front-ends that interface the device with transmitter or receiver antennas. We use LFTX and LFRX daughterboards, that operate from DC to $30 \mathrm{MHz}$, which includes ultrasonic frequency ranges of interest to us.

In USRP, the system complexity is shifted from hardware to software and most of the computational load is typically left to the host machine. However, recent literature [15], [16] has shown that the host machine can become the computational bottleneck of the communication system, while the connection between host and USRP introduces delays preventing accurate timing of network protocols. As discussed in detail in what follows, we overcome this problem by shifting (with respect to the typical GNU Radio/USRP architecture) significant components of the signal processing on the on-board FPGA.

Host Machine. The host machine can be either a desktop/laptop computer or a computer-on-module, e.g., Gumstix, connected to the USRP through a Gigabit Ethernet $(\mathrm{GbE})$ link. In the traditional GNU Radio/USRP architecture, the host machine runs all the software-defined signal processing functionalities implemented with the GNU Radio development toolkit [17]. However, for reasons discussed in detail in Section IV, we chose to implement most PHY and MAC functionalities in the FPGA embedded in the USRP. Therefore, in our design the host machine only configures and initializes the USRP, and generates/receives application-layer bit streams.

Electronic Switch. To reduce the testbed complexity and cost, we use an electronic switch that allows a single ultrasonic transducer to transmit and receive on a time division basis. The switching operation is piloted both from the host machine and the USRP FPGA by connecting the switch with the General Purpose Input/Output (GPIO) digital pins available on the LFTX and LFRX daughterboards. We use a commercial offthe-shelf (COTS) switch, Mini-Circuits ZX80-DR230+ [18], that comes in a connectorized package with embedded coaxial RF connectors, and offers low insertion loss and very high isolation over the entire frequency range $(0-3 \mathrm{GHz})$.

Amplification Stage. We introduced an external amplification stage. The low-power output of the LFTX daughterboards, about $3 \mathrm{dBm}(\approx 2 \mathrm{~mW})$, can limit the maximum transmission range supported. Therefore, at the transmitter we use a connectorized COTS Power Amplifier (PA), Mini-Circuits ZPUL- 
30P [18], specifically designed for short-pulse transmissions with a maximum output power of $22 \mathrm{dBm}$. In the receiver chain, LFRX daughterboards have almost no gain. Thus, we use a connectorized COTS Low-Noise Amplifier (LNA), MiniCircuits ZFL-1000LN+ [18], with a noise figure of $2.9 \mathrm{~dB}$.

Ultrasonic Transducers. An ultrasonic transducer is a device capable of transmitting and receiving ultrasonic waves. Most commercial ultrasonic transducers are based on the piezoelectric effect, which allows converting electrical energy in ultrasonic energy, and vice versa [19].

As discussed in Section II-A, to communicate in human tissues over a range of several centimeters we need transducers operating at frequencies in the order of a few $\mathrm{MHz}$. Moreover, high-bandwidth transducers are necessary to implement wideband transmission schemes such as UsWB. We found that the only COTS ultrasonic transducers that nearly match our requirements are those designed for nondestructive analysis (NDA) applications [20], since high frequencies and large bandwidth are required for fine material characterization. However, these transducers are not optimized in terms of coupling electromechanical efficiency, and thus introduce significant energy conversion losses. Moreover, NDA transducers are characterized by high directivity. In our current testbed we use standard immersion W-series ultrasonic transducers, Ultran WS37-5 [20]. The nominal bandwidth central frequency is about $5 \mathrm{MHz}$ and the bandwidth at $-6 \mathrm{~dB}$ goes from $50 \%$ to $100 \%$ of the bandwidth central frequency, i.e., $2.5-5 \mathrm{MHz}$.

Ultrasonic Phantoms. We use ultrasonic phantoms to emulate the intra-body ultrasonic communication channel with high fidelity [21]. Commonly employed in medical ultrasound research, ultrasonic phantoms are composed of soft and hard tissue-mimicking materials, also known as tissue substitutes. These materials have the same acoustic propagation properties of human tissues, e.g., sound speed, density, and attenuation. Off-the-shelf ultrasonic phantoms that emulate the interactions between ultrasounds and the human body, tissues, organs and systems are available [22].

We selected a human-kidney phantom immersed in a background water-based gel [22], whose acoustic characteristics are reported in Table I. The background gel is almost lossless, and has the same density and sound speed as the kidney. Therefore, reflections and refractions are minimum between the kidney and the gel. Thus, the latter can be considered acoustically transparent. The phantom dimensions are approximately $10 \times 16 \times 20 \mathrm{~cm}$.

TABLE I

ULTRASONIC PHANTOM ACOUSTIC CHARACTERISTICS

\begin{tabular}{cccc}
\hline \hline Tissue & Speed, $v$ & Attenuation, $\alpha$ & Density, $\rho$ \\
\hline Background Gel & $1550 \mathrm{~m} / \mathrm{s}$ & $<0.1 \mathrm{~dB} / \mathrm{cm}$ & $1020 \mathrm{Kg} / \mathrm{m}^{3}$ \\
Kidney & $1550 \mathrm{~m} / \mathrm{s}$ & $2 \mathrm{~dB} / \mathrm{cm} \mathrm{@} 5 \mathrm{MHz}$ & $1030 \mathrm{Kg} / \mathrm{m}^{3}$ \\
\hline
\end{tabular}

\section{Architecture of the Communication System}

Signal processing, algorithms and protocols can be implemented in the ultrasonic software-defined node using a framework that combines (i) the GNU Radio software development toolkit [17] and (ii) the open source Hardware Description Language (HDL) design for the FPGA embedded in the USRP. In GNU Radio, most of the digital signal processing is performed on the external host. When using HDL design most signal processing operations are moved to the embedded FPGA. In this Section we discuss the tradeoffs between these two different approaches.

\section{A. GNU Radio Vs. HDL PHY Layer Implementation}

As discussed in Section II-B, wideband pulse-based communications can significantly mitigate the multipath effect caused by the heterogeneity of the human body. However, shorter pulses have wider bandwidth, which results in higher sampling rates that can overload the host machine or the Gigabit Ethernet $(\mathrm{GbE})$ link between the host machine and the USRP. In our initial design, UsWB PHY layer functionalities were implemented on the host machine using GNU Radio. However, we observed the following limitations: (i) the capacity of the GbE link between the host machine and the USRP limits the maximum achievable sample rate, i.e., 25 million samples per second, thus the maximum achievable signal bandwidth. When exceeding the link capacity, Ethernet frames coming from/to the USRP are dropped at the network interfaces, with consequent loss of the carried digital samples; (ii) digital signal processing operations implemented in GNU Radio, e.g., digital filters, overload the host machine when operating at high sampling rates, i.e., greater than 10 million samples per second. If the host machine is unable to process data fast enough, the internal buffers that store digital samples overflow, thus causing loss of large amount of digital samples.

It became apparent that the above limitations would prevent successful implementation of UsWB in GNU Radio. For these reasons, we chose to implement all PHY layer functionalities in the embedded FPGA. This effectively speeds up data processing and reduces the computational load on the host machine.

Partial Reconfiguration. The price we pay for these benefits is a lower system flexibility. The HDL design needs to be synthesized before it can be loaded in the embedded FPGA. Thus, changing the PHY layer structure and parameters at runtime is not as simple as doing it in GNU Radio on the host machine. Still, we designed the HDL modules such that partial PHY layer reconfiguration is achievable at runtime through a group of setting registers implemented on the FPGA that can be accessed by the host machine. Through these setting registers, one can reconfigure key parameters of the PHY layer transmission scheme (i.e., pulse shape and code length, among others) or even select which PHY blocks should be used, thus modifying at runtime the structure of the PHY layer chain.

\section{B. MAC Layer Design Challenges}

Similarly, MAC-layer functionalities can potentially be implemented in the host machine or in the embedded FPGA. Highly customized or reconfigurable and complex protocols can be challenging to implement on FPGA and likely cause overutilization of the available FPGA hardware resources. An 


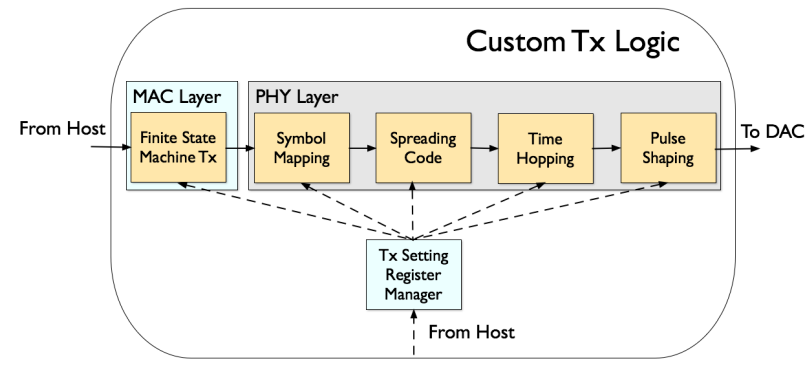

Fig. 3. Block scheme of the custom transmitter logic.

appealing alternative is then to implement the MAC layer on the host machine, by using high-level languages and libraries available in GNU Radio. However, MAC protocols require highly precise packet timing and small, precise interframe spacings in the order of microseconds. We observed that GbE link and the GNU Radio processing latency are in the order of milliseconds [16]. Hence, time-critical radio or MAC functions cannot be placed in the host machine.

In the current system architecture the MAC layer is implemented in HDL, and the FPGA setting registers enable partial reconfiguration at runtime. We are currently working on the implementation of a hybrid solution [16], [23] based on a softcore processor implemented in the embedded FPGA.

\section{TX AND RX HDL ARCHITECTURE}

The default USRP HDL design operates on digital waveforms coming from and going to the host machine and performs only digital down and up conversion (DDC/DUC), decimation and interpolation. PHY and MAC layer digital processing takes place on the host machine. We followed a different approach, and we customized and extended the USRP HDL code to implement UsWB PHY and MAC layer operations in the FPGA.

\section{A. Custom Transmitter Chain}

Figure 3 shows a block diagram of the custom transmitter logic. Since PHY and MAC functionalities are implemented on the FPGA, input data are raw information bits that need to be packetized and encoded in digital waveforms. After MAC and PHY layer operations, the custom transmitter logic outputs the digital quantized signal to be transmitted. This is then digitalto-analog converted, amplified and finally converted into an ultrasonic signal by the transmitter transducer.

Transmitter MAC Finite State Machine. Raw information bits from the host are received at the transmitter MAC-layer Finite State Machine (FSM-Tx), which implements UsWB MAC functionalities (including packetization) and coordinates the PHY layer operations implemented by the other blocks of the chain. The UsWB MAC-layer data structure, i.e., UsWB packet ${ }^{1}$ is created in the FSM-Tx. The UsWB packet starts after a Packet Synchronization Preamble (PSP) and a Time-Hopping Synchronization Preamble (THSP). The former enables coarse synchronization that allows the receiver to

\footnotetext{
${ }^{1}$ We intentionally use the word packet instead of frame to avoid confusion with the time-hopping frame in Section II-B.
}
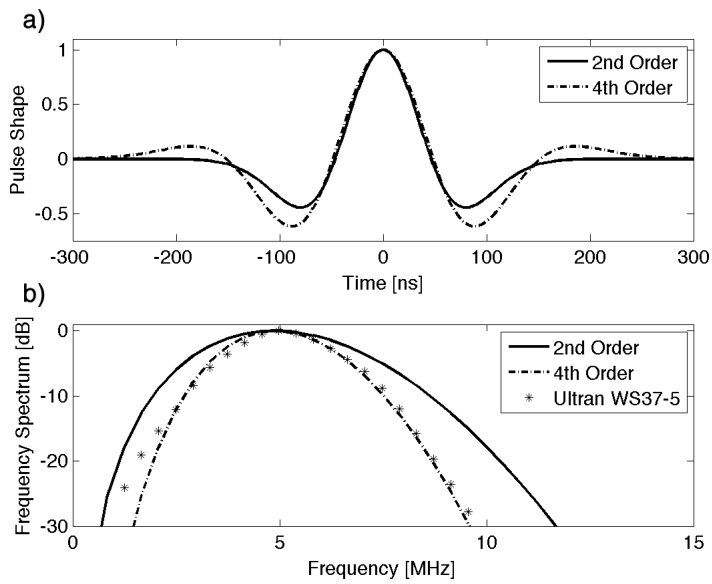

Fig. 4. a) 2nd-order and 4th-order derivative gaussian pulses, b) compared in frequency domain with the Ultran WS37-5 frequency response.

detect an incoming packet, while the latter allows identifying the exact start time of the time-hopping frame. The UsWB packet is then serialized, i.e., converted into a sequence of bits, and forwarded to the next module in the chain, i.e., Symbol Mapping. The FSM-Tx also controls the time-hopping frame length $N_{h}$ and spreading code length $N_{s}$ used by the PHY layer, according to feedback information from the receiver.

Transmitter PHY Layer. The first block of the transmitter PHY layer chain is Symbol Mapping. Here, raw information bits are mapped into $\{-1,1\}$ binary symbols. The binary symbols are then spread in chips by the Spreading Code module following a pseudo-random spreading code. For each symbol, this block outputs $N_{s}$ chips in $\{-1,1\}$. Chips are then forwarded to the Time-Hopping module that spreads them in time according to the selected time-hopping pattern. The output of this block is a sequence of $\{-1,1\}$ chips, one per time-hopping frame. Finally, the Pulse Shaping module maps the incoming chips to position-modulated pulses. The output is a train of position-modulated pulses following a predefined time-hopping pattern, as described in (2).

Pulse Shaping. The Pulse Shaping module consists of a Finite Impulse Response (FIR) filter whose coefficients, i.e., taps, represent the samples of a 4th-order derivative gaussian pulse. The original design discussed in [2] was based on a 2nd-order derivative gaussian pulse. However, to match the central frequency and bandwidth requirements of the ultrasonic transducers in use, we adopted a higher-order derivative gaussian pulse characterized by higher central frequency and lower relative bandwidth [24]. In Fig. 4a and Fig. 4b the two pulse shapes are compared in the time and frequency domains. Figure $4 \mathrm{~b}$ also shows a 4th-order derivative pulse shaped to match the frequency response of the Ultran WS375 ultrasonic transducer. The pulse duration is approximately $300 \mathrm{~ns}$, with a PPM shift of $60 \mathrm{~ns}$, within a time-hopping slot ( $T_{c}$ in Section II-B) of $360 \mathrm{~ns}$. The resulting maximum raw chip rate is $2.78 \mathrm{Mchip} / \mathrm{s}$, which results in a maximum raw data rate of $2.78 \mathrm{Mbit} / \mathrm{s}$ when time-hopping frame and spreading code lengths are both set to 1 . 


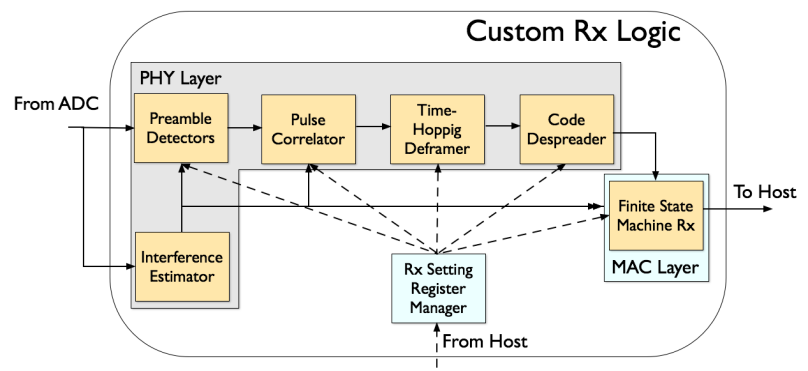

Fig. 5. Block scheme of the custom receiver logic.

Transmitter Setting Register Manager. The Transmitter Setting Register Manager (SRM-Tx) is in charge of routing configuration parameters written by the host machine into the setting registers discussed in Section IV-A. Whenever the host machine updates any of the setting registers, the SRM-Tx is triggered to read the register content and route it to the destination module. This block enables real-time reconfiguration thus enhancing the transmitter flexibility.

The communication system is designed to allow real-time reconfiguration of several parameters, i.e., spreading code and spreading code length, time-hopping frame length and timehopping sequence, packet payload size, SPD sequence and SPD length, and length of the preambles. One can also change the pulse shape in real time through FIR filters with reloadable taps. Moreover, by carefully rerouting the binary flow, we can use the registers to enable and disable selected modules to reconfigure in real time the entire chain structure, thus modifying the communication system architecture. For example, we can implement adaptive modulation by switching between different Symbol Mapping modules at runtime to change the symbol constellation used. Moreover, we can disable the TimeHopping module or the Spreading Code module to obtain pure spreading-code or time-hopping based schemes.

\section{B. Custom Receiver Chain}

The custom receiver chain, illustrated in Fig. 5, implements receiver UsWB PHY and MAC layer functionalities. The received ultrasonic signal is converted to an electrical signal by the RX transducer. The signal is amplified by the LNA, and analog-to-digital converted by the USRP ADC. Then, the digital waveform is processed by the custom receiver chain in the FPGA. After PHY and MAC operations, the custom receiver chain outputs a binary stream representing the received decoded data.

Receiver MAC Finite State Machine. The Receiver MAC Finite State Machine (FSM-RX) implements UsWB MAC protocol functionalities and coordinates the PHY-layer logic. The FSM-Rx detects the received packet based on information coming from preamble detectors, and triggers the PHY layer module to start processing the received waveform. Finally, it decodes the received bits based on the output of the PHY layer operations on the received digital waveforms. Moreover, FSMRx estimates the level of interference, and accordingly chooses the optimal pair of time-hopping frame length and spreading code length, i.e., those that maximize the communication throughput while keeping the bit error rate (BER) under a predefined threshold - see [2] for details.

Preamble Detectors. The preamble detectors are designed to achieve packet and time-hopping synchronization. The former enables coarse synchronization by identifying the presence of an incoming packet. The latter identifies the exact start point of the time-hopping frame.

The Packet Synchronization Preamble (PSP) consists of a train of pulses positioned in consecutive time slots. The PSP detector includes a single-rate FIR filter used as a correlator, a squaring module, an integrator, and a threshold-based plateau detector. The FIR filter is used to correlate the incoming pulses with a 5th-order derivative gaussian pulse, the filter impulse response. Squaring and integrating the correlator output ideally results in a constant output for the whole PSP duration, i.e., a plateau. Therefore, the packet can be coarsely detected by finding the plateau. By using a dynamic threshold adaptation, this procedure can be made independent of the noise floor.

Fine synchronization is performed by the Time-Hopping Synchronization Preamble (THSP) detector, which includes a single-rate FIR filter used as correlator, and a threshold-based correlation peak detector. The THSP consists of a train of pulses, each positioned in the first time slot of consecutive time-hopping frames. By correlating the THSP with a 5thorder derivative gaussian pulse, we obtain a peak in the first slot of each time-hopping frame. The beginning of the timehopping frame is determined by the threshold-based peak detector. Again, the threshold is dynamically adapted to the noise floor level.

Receiver PHY Layer. The receiver PHY layer module implements the bit decoding operations that can be formally expressed as

$$
\sum_{j=0}^{N_{s}-1} a_{j} \int_{c_{j} T_{c}-j T_{f}}^{\left(c_{j}+1\right) T_{c}-j T_{f}} s(t) \cdot c(t) d t \underset{d_{1}}{\stackrel{d_{-1}}{\gtrless}} 0,
$$

where $c(t)$ is the correlator function, while all the other symbols follow the signal model presented in Section II-B.

The first module in the receiver PHY chain is the Pulse Correlator, i.e., a decimator FIR filter with a 5th-order derivative gaussian pulse impulse response. The Pulse Correlator outputs one sample per time slot, and the FIR impulse response, i.e., the correlator function $\mathrm{c}(\mathrm{t})$, is selected in such a way as to give a zero output for an empty slot, a positive value for a slot containing a '-1' chip, and a negative value for a slot containing a ' 1 ' chip. The correlation output goes into the Time-Hopping Deframer, which collects the non-zero inputs located according to the Time-hopping sequence used at the transmitter. The Time-Hopping Deframer determines the integral intervals and the Pulse Correlator performs the actual integration in (3). Finally, the Code Despreader inverts the spreading operation by weighting the correlation output with the spreading code originally used at the transmitter and summing these over the spreading code length. This operation corresponds to the weighted sum in (3). Based on the result of the despreading operation, the FSM-Rx makes a decision on 


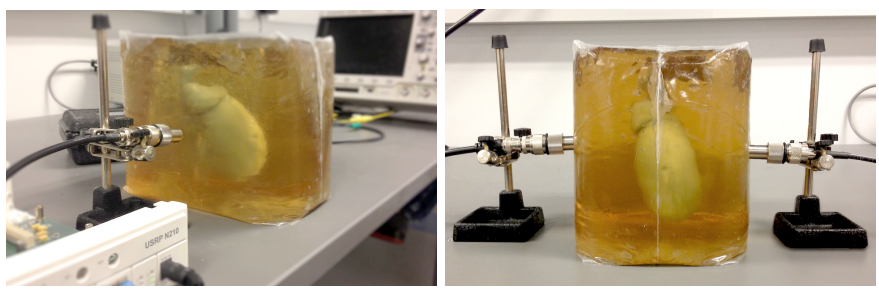

Fig. 6. Two nodes communicating through a human-kidney phantom.

the received bits. If the resulting sum is positive a '-1' symbol is received $\left(d_{-1}\right)$, otherwise a ' 1 ' symbol is received $\left(d_{1}\right)$.

Receiver Setting Register Manager. The Receiver Setting Register Manager (SRM-Rx) provides the same functionalities as the SRM-Tx. The SRM-Rx operates on registers different than those used in the transmission chain. Therefore, the transmitter and receiver chains of each node can be independently reconfigured in real time.

Interference Level Estimation. The Interference Level Estimation module is needed by the UsWB MAC protocol to perform the rate-maximizing adaptation described in detail in [2]. The level of interference is estimated in terms of number of interfering pulses per time-hopping frame. The estimation module consists of a single-rate FIR filter whose impulse response is a 5th-order derivative gaussian pulse, a squaring module, an integrator and a peak detector with dynamically adapted threshold. By comparing at each time slot the integrator output with the threshold, we can detect the presence of pulses, and therefore how many pulses are received in a time-hopping frame. The level of interference is obtained by averaging over the entire packet reception time.

\section{UsWB Performance Evaluation}

In this section we demonstrate the feasibility of ultrasonic intra-body communications through testbed experiments. We start by evaluating the physical layer performance of the prototype we developed in terms of BER by varying the transmit power, the time-hopping frame and spreading code length and the level of interference in the channel. Then, we show how MAC adaptation allows a pair of nodes to adapt the communication rate according to the level of ultrasonic interference, to maximize the throughput while satisfying packet drop rate reliability constraints.

\section{A. UsWB PHY Layer}

The testbed setup consists of two ultrasonic softwaredefined nodes communicating through a human-kidney phantom (Fig. 6). The two nodes ultrasonic transducers are positioned in the opposite sides of the phantom smaller dimension, i.e., $10 \mathrm{~cm}$, and the kidney is centered in the background gel such to be aligned between the two ultrasonic transducers. To guarantee repeatability of the experiments, we generate interference from co-located transceivers artificially by injecting interfering pulses at the transmitter. The position of the interfering pulses inside the time-hopping frame is given by a pseudo-random generator HDL module, i.e., a Linear Feedback Shifter Register (LFSR).

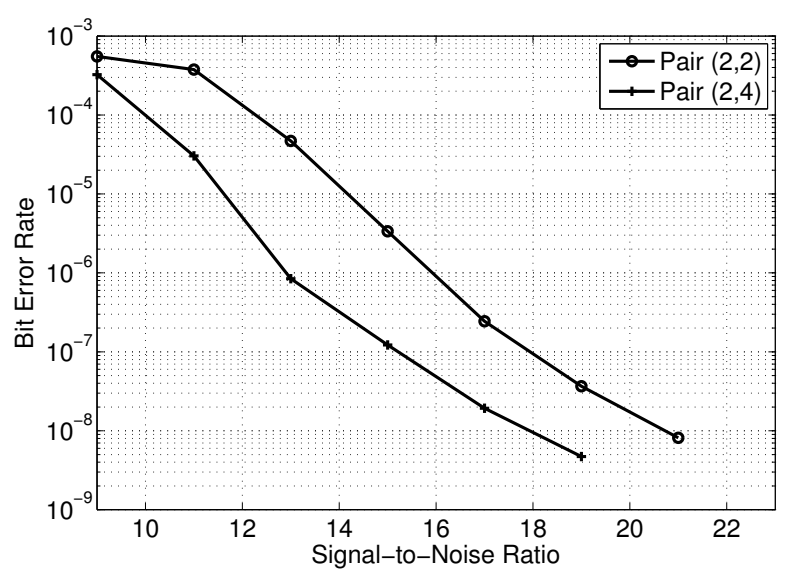

Fig. 7. BER in the absence of interference as a function of the SNR.

BER Vs SNR. First, we evaluate the BER as a function of the SNR per pulse measured at the receiver, in the absence of external interference, with fixed time-hopping frame and spreading code length. We define the SNR per pulse as the ratio between the received energy per pulse and the noise power spectral density. By connecting a variable-gain attenuator between the LFTX daughterboard and the power amplifier we vary the input power at the Tx transducer between $-7 \mathrm{dBm}$ and $-21 \mathrm{dBm}$, to obtain values of SNR between 23 and $9 \mathrm{~dB}$, respectively. In Fig. 7, the resulting BER is depicted for time-hopping frame length and spreading code length pairs $(2,2)$ and $(2,4)$. We observe that, as expected, the BER is a decreasing function of the SNR and that by using longer spreading code the BER is further reduced. If we further decrease the SNR at the receiver, i.e., to $7 \mathrm{~dB}$ (a transmit power of $-23 \mathrm{dBm}$ ), communication fails altogether due to limitations in the current time synchronization scheme. In the considered "kidney" setup, the UsWB prototype achieves $347.21 \mathrm{kbit} / \mathrm{s}$ with a $10^{-6}$ BER at $13 \mathrm{~dB}$ SNR, which corresponds to an input power at the Tx transducer of about $-17 \mathrm{dBm}(\approx 20 \mu \mathrm{W})$. A data rate up to about $700 \mathrm{kbit} / \mathrm{s}$ can be achieved (also with $10^{-6}$ BER) with a $(2,2)$ pair increasing the input power to $-14 \mathrm{dBm}(\approx 40 \mu \mathrm{W})$, i.e., $16 \mathrm{~dB}$ SNR. Lower-power transmissions are also possible by compensating with longer spreading code. For example, in the current implementation, for a Tx power of $-21 \mathrm{dBm}(\approx 8 \mu \mathrm{W})$, i.e., $9 \mathrm{~dB}$ SNR, and with a spreading code of 20 chips, we obtain a data rate of $70 \mathrm{kbit} / \mathrm{s}$ with a BER lower than $10^{-6}$.

Energy conversion losses can be reduced with customdesigned ultrasonic transducers with higher coupling electromechanical efficiency to further reduce the Tx power requirements. Moreover, we acknowledge that there is significant room for improving the current time synchronization scheme, after which it will be possible to operate at even lower SNRs.

BER Vs Time-Hopping and Spreading Code. In Fig. 8 (top), we evaluate the BER by varying the time-hopping frame and spreading code length with 4 interfering pulses per frame. Since we focus on the effect of the interference, we set the the input power at the Tx transducer to $13 \mathrm{dBm}$, to create 

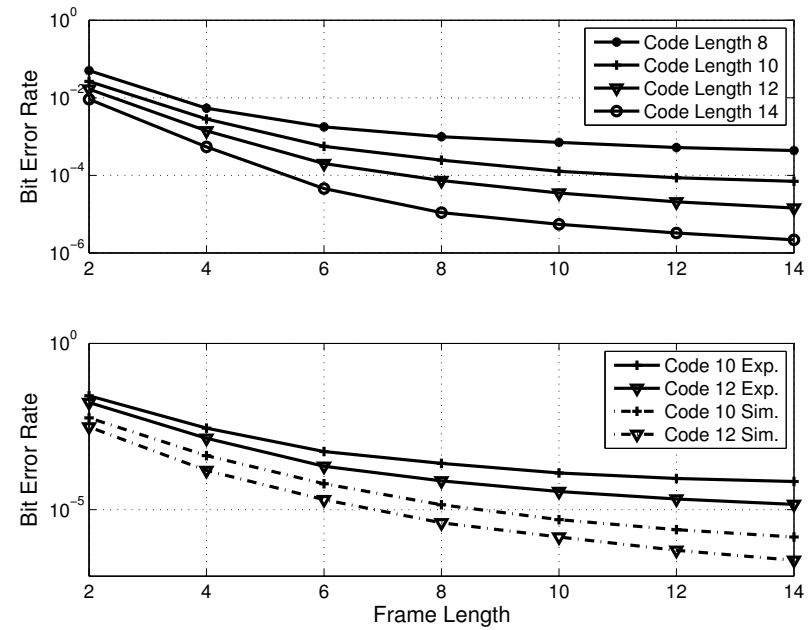

Fig. 8. BER as a function of frame length, with 4 interfering pulses per frame for different values of code length (top), compared with simulated BER (bottom).

high-SNR condition, i.e., 43 dB SNR. In Fig. 8 (bottom) the experimental results are compared with BER curves obtained by simulating the same scenario with the UsWB PHY layer simulator described in [2]. For fairness, the pulse shape used in the simulator is obtained by recording a real pulse shape as received in testbed experiments. Therefore, we consider the signal distortion introduced by amplifiers and transducers, and the scattering and reflection effects introduced by the ultrasonic phantom. However, the imported deterministic measurement does not consider the time-variability of the real testbed conditions, e.g., operating temperature, humidity, and coupling of the transducer with the phantom surface, among others. We observe that, as expected, the BER is a decreasing function of the time-hopping frame length and the spreading code length, thus confirming the simulation results in our previous work [2].

\section{B. UsWB MAC Layer}

We consider a pair of ultrasonic software-defined nodes and evaluate how the UsWB MAC protocol adapts the link parameters to compensate for varying levels of interference, i.e., multiple concurrent transmissions. The level of interference is defined in terms of number of interfering pulses within a time-hopping frame. If we assume that all nodes measure the same level of interference, that is, all network nodes are close enough to be all in the same transmission range, i.e., as in the implicitly cooperative problem in [2], the number of interfering pulses per time-hopping frame coincides with the number of co-located active Tx-Rx pairs.

UsWB Rate-Maximizing Adaptation. First, we evaluate the UsWB MAC rate-maximizing adaptation as a function of the level of interference. We transmit 250 packets and increase the level of interference every 50 packets, from zero interfering pulses per time-hopping frame to four. In Fig. 9 (bottom), we show the estimated level of interference at the receiver. We observe that the receiver occasionally overestimates the number of interferers, which however does
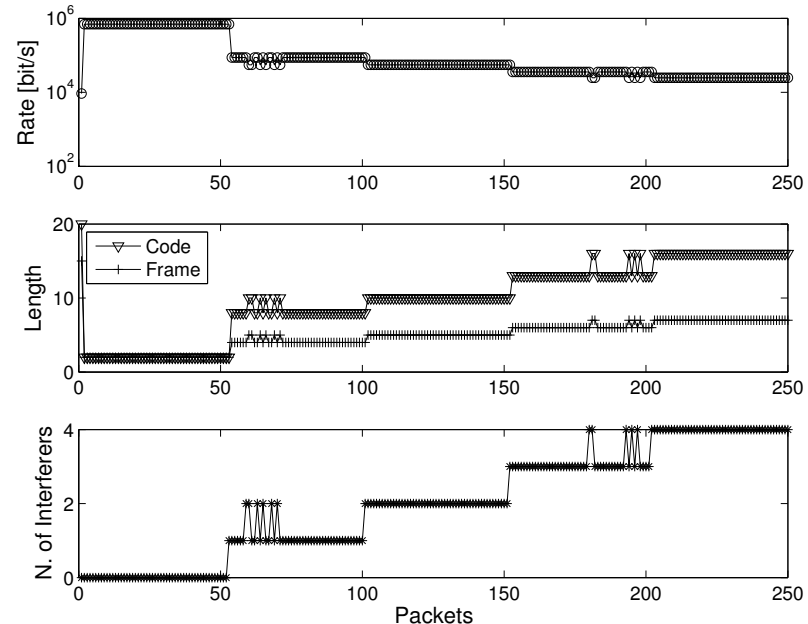

Fig. 9. Rate-maximizing adaptation. Interference estimate (top), optimal time-hopping frame and spreading code length (middle) and data rate of the transmitter (bottom).
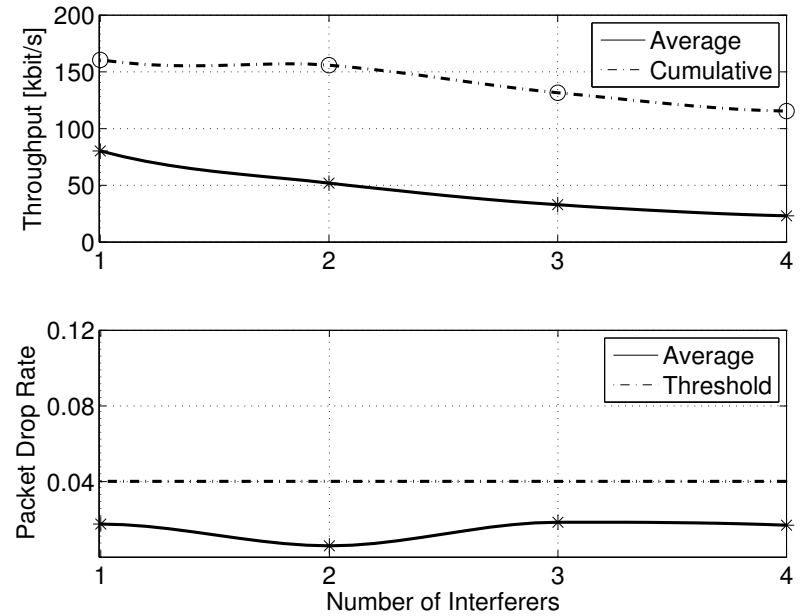

Fig. 10. Throughput (top) and packet drop rate (bottom) as a function of the number of interferers.

not affect the performance in terms of packet drop rate. According to the UsWB protocol, based on the interference estimation, the receiver computes the optimal pair of timehopping frame and spreading code length, and piggybacks these in ACK/NACK packets. In the current implementation, the optimization problem in [2] is solved offline, and the solution is then loaded into the receiver. By performing a lookup table operation, the receiver finds the optimal pair corresponding to the measured level of interference and BER requirements. Figure 9 (middle) shows the time-hopping frame and spreading code length used by the transmitter after the ACK/NACK is received. As expected, these vary according to the interference estimate at the receiver. The resulting data rate of the transmitter is shown in Fig. 9 (top).

Throughput and Packet Drop Rate. To verify the effectiveness of the rate adaptation, we evaluate throughput and packet drop rate at the receiver while varying the level of interference. We define throughput as the average bit rate of correctly received information during a time window. The 

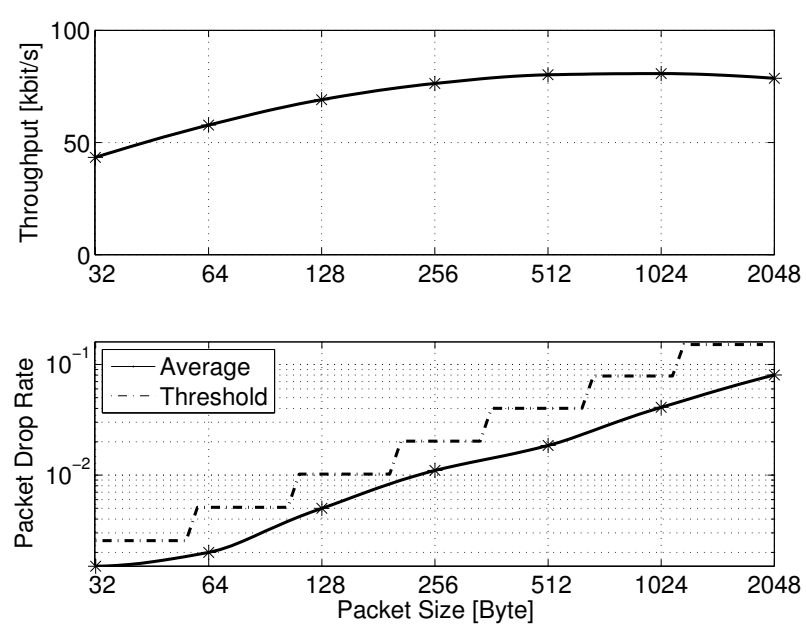

Fig. 11. Throughput (top) and packet drop rate (bottom) as a function of the packet size with one co-located interfering Tx-Rx pair.

packet drop rate is defined as the ratio between the number of packets dropped and the number of packets generated at the application layer. We set the packet payload length to 512 bytes and vary the level of interference form zero to four every 5000 packets. In Fig. 10 (top), we show throughput of a single Tx-Rx pair along with the cumulative throughput considering all the concurrent interfering Tx-Rx pairs. Figure 10 (bottom) shows the resulting packet drop rate compared with the maximum packet drop rate threshold (4\%) corresponding to the maximum BER constraint $\left(<10^{-5}\right)$ and packet size.

Optimal Packet Size. Finally, we investigate how the packet size affects throughput and packet drop rate. We set the number of interferers to one, and transmit a fixed amount of data ( $\approx 2$ MBytes) with different packet sizes. In Fig. 11a, we observe that the resulting throughput is maximized between 512 and 1024 bytes. The optimal packet size is influenced by the packet drop rate and protocol overhead. For a given BER, the packet drop rate is determined by the packet size, i.e., the longer the packet the higher the packet drop rate. At the same time, longer packets result in less protocol overhead (packet header, ACK/NACK control packets, and propagation delay). Figure 11b shows that the packet drop rate increases when the payload size increases. However, the maximum packet drop rate constraint is always satisfied.

\section{CONCLUSIONS}

We discussed design and implementation of a softwaredefined testbed architecture for ultrasonic intra-body area networks, and experimentally demonstrated for the first time the feasibility of ultrasonic communications in biological tissues. We discussed our prototype implementation and showed that our prototype can flexibly trade data rate performance for power consumption. Finally, we showed how the considered MAC protocol allows multiple transmitter-receiver pairs to coexist and dynamically adapt the transmission rate according to the channel and the level of interference condition, to maximize the throughput while satisfying predefined reliability constraints.

\section{REFERENCES}

[1] L. Galluccio, T. Melodia, S. Palazzo, and G. E. Santagati, "Challenges and Implications of Using Ultrasonic Communications in Intra-body Area Networks," in Proc. of IEEE Intl. Conf. on Wireless On-demand Networked Systems (WONS), Courmayeur, Italy, Jan. 2012.

[2] G. E. Santagati, T. Melodia, L. Galluccio, and S. Palazzo, "Distributed MAC and Rate Adaptation for Ultrasonically Networked Implantable Sensors," in Proc. of IEEE Conf. on Sensor, Mesh and Ad Hoc Communications and Networks (SECON), New Orleans, LA, June 2013.

[3] B. Latre', B. Braem, I. Moerman, C. Blondia and P. Demeester, "A Survey on Wireless Body Area networks," ACM/Springer Wireless Networks, vol. 17, no. 1, pp. 1-18, Jan. 2011.

[4] E. Chow, S. Chakraborty, W. Chappell, and P. Irazoqui, "Mixed-signal integrated circuits for self-contained sub-cubic millimeter biomedical implants," in IEEE Intl. Conf. on Solid-State Circuits Conference (ISSCC), Feb. 2010, pp. $236-237$.

[5] T. Melodia, H. Kulhandjian, L. Kuo, and E. Demirors, "Advances in Underwater Acoustic Networking," in Mobile Ad Hoc Networking: Cutting Edge Directions, second edition ed., S. Basagni, M. Conti, S. Giordano, and I. Stojmenovic, Eds. Inc., Hoboken, NJ: John Wiley and Sons, 2013, pp. 804-852.

[6] F.L. Thurstone, H.E. Melton, "Biomedical Ultrasonics," IEEE Trans. on Ind. Electr. and Contr. Instrum., vol. 17, no. 2, Apr. 1970.

[7] G. Lockwood, D. Turnball, D. Christopher, and F. Foster, "Beyond $30 \mathrm{MHz}$ [applications of high-frequency ultrasound imaging]," IEEE Engineering in Medicine and Biology Magazine, vol. 15, no. 6, Nov/Dec 1996.

[8] R. Smith, A. Arca, X. Chen, L. Marques, M. Clark, J. Aylott, and M. Somekh, "Design and fabrication of nanoscale ultrasonic transducers," Journal of Physics: Conference Series, vol. 353, no. 1, 2012.

[9] A. Y. Cheung and A. Neyzari, "Deep local hyperthermia for cancer therapy: External electromagnetic and ultrasound techniques," Cancer Res. (Suppl.), vol. 44, no. 9, 1984.

[10] T. Hogg and R. A. Freitas, "Acoustic Communication for Medical Nanorobots," Nano Communication Networks (Elsevier), vol. 3, no. 2, pp. 83-102, Feb. 2012.

[11] C. R. Hill, Ultrasonic attenuation and scattering by tissues. John Wiley \& Sons, Inc., 1978.

[12] F. P. Bolin, L. E. Preuss, R. C. Taylor, and R. J. Ference, "Refractive index of some mammalian tissues using a fiber optic cladding method," Appl. Opt., vol. 28, no. 12, pp. 2297-2303, Jun 1989.

[13] USRP: Universal Software Radio Peripheral. [Online]. Available: http://www.ettus.com/

[14] WARP: Wireless Open-Access Research Platform, Rice University. [Online]. Available: http://warp.rice.edu/index.php

[15] G. Nychis, T. Hottelier, Z. Yang, S. Seshan, and P. Steenkiste, "Enabling MAC protocol implementations on software-defined radios," in Proc. of USENIX symposium on Networked systems design and implementation, Boston, MA, 2009.

[16] K. Chowdhury and T. Melodia, "Platforms and Testbeds for Experimental Evaluation of Cognitive Ad Hoc Networks," IEEE Communications Magazine, vol. 48, no. 9, pp. 96-104, Sep 2010.

[17] GNU Radio - The GNU Software Radio. [Online]. Available: http://www.gnu.org/software/gnuradio/

[18] Mini-Circuits: RF/IF \& Microwave components DC to $40 \mathrm{GHz}$. [Online]. Available: http://www.minicircuits.com/

[19] K. Shung and M. Zippuro, "Ultrasonic transducers and arrays," IEEE Engineering in Medicine and Biology Magazine, vol. 15, no. 6, pp. 20 -30 , nov 1996.

[20] Standard Immersion Ultrasonic Transducers. [Online]. Available: http://www.ultrangroup.com/index.php/products/transducers/standard/

[21] M. O. Culjat, D. Goldenberg, P. Tewari, and R. S. Singh, "A review of tissue substitutes for ultrasound imaging," Ultrasound in Medicine \& Biology, vol. 36, no. 6, pp. $861-873,2010$.

[22] CIRS: Kidney Training Phantom. [Online]. Available: http://www.cirsinc.com/products/all/81/kidney-training-phantom/

[23] K. Tan, H. Liu, J. Zhang, Y. Zhang, J. Fang, and G. M. Voelker, "Sora: high-performance software radio using general-purpose multicore processors," Commun. ACM, vol. 54, no. 1, pp. 99-107, Jan. 2011.

[24] M. Di Benedetto, Ultra-wideband Communication Systems: A Comprehensive Overview, ser. EURASIP Book Series on Signal Processing and Communications. Hindawi Publishing Corporation, May 2006. 